\title{
The inhibitory roles of Ihh downregulation on chondrocyte growth and differentiation
}

\author{
ANG DENG ${ }^{1}$, HONGQI ZHANG ${ }^{1}$, MINYU HU ${ }^{2}$, SHAOHUA LIU ${ }^{1}$, \\ YUXIANG WANG $^{1}$, QILE GAO $^{1}$ and CHAOFENG GUO ${ }^{1}$ \\ ${ }^{1}$ Department of Spine Surgery, Xiangya Hospital of Central South University, \\ Changsha, Hunan 410008; ${ }^{2}$ Department of Nutrition and Food Hygiene, School of Public Health, \\ Central South University, Changsha, Hunan 410078, P.R. China
}

Received February 24, 2017; Accepted November 3, 2017

DOI: $10.3892 / e t m .2017 .5458$

\begin{abstract}
The proliferative rate of chondrocytes affects bone elongation. Chondrocyte hypertrophy is required for endochondral bone formation as chondrocytes secrete factors required for osteoblast differentiation and maturation. Previous studies have demonstrated that the Indian hedgehog (Ihh) signaling pathway is a key regulator of skeletal development and homeostasis. The aim of the present study was to investigate the function of Ihh in chondrocyte proliferation and differentiation, as well as the underlying mechanisms. Ihh was knocked down in mouse chondrocyte cells using short hairpin RNA. Chondrocyte apoptosis and cell cycle arrest were assessed using flow cytometry and the results indicated that knockdown of Ihh significantly inhibited cell growth $(\mathrm{P}<0.05)$ and increased apoptosis $(\mathrm{P}<0.001)$ compared with negative control cells. Downregulation of Ihh also resulted in cell cycle arrest at G1 to S phase in chondrocytes. It was also observed that knockdown of Ihh decreased alkaline phosphatase activity and mineral deposition of chondrocytes. The inhibitory roles of Ihh downregulation on chondrocyte growth and differentiation may be associated with the transforming growth factor- $\beta /$ mothers against decapentaplegic and osteoprotegerin/receptor activator of nuclear factor $\kappa \mathrm{B}$ ligand signaling pathway. The results of the present study suggest that chondrocyte-derived Ihh is essential for maintaining bone growth plates and that manipulation of Ihh expression or its signaling components may be a novel therapeutic technique for the treatment of skeletal diseases, including achondroplasia.
\end{abstract}

Correspondence to: Dr Shaohua Liu, Department of Spine Surgery, Xiangya Hospital of Central South University, 87 Xiangya Road, Changsha, Hunan 410008, P.R. China

E-mail: liushaohua_1@163.com

Key words: Indian hedgehog, chondrocyte, differentiation, cell cycle, apoptosis, endochondral bone formation

\section{Introduction}

Bone formation in the developing embryo is governed by intramembranous and endochondral ossification initiating from mesenchymal condensations, in which mesenchymal progenitor cells differentiate (1). During endochondral ossification, mesenchymal progenitors differentiate into chondrocytes to form the cartilage template of the bone, which is required for bone elongation. Chondrocyte hypertrophy is required for endochondral bone formation; elongated chondrocytes exit the cell cycle to become prehypertrophic chondrocytes prior to terminally differentiating to form hypertrophic chondrocytes (2). These chondrocytes subsequently secrete factors required for osteoblast differentiation and maturation (3).

It has previously been reported that the Indian hedgehog (Ihh) signaling pathway is a key regulator of skeletal development and homeostasis (4) and that Ihh signaling must be enhanced to increase bone formation (5). In a previous study, overexpression of Ihh ameliorated short stature homeobox 2 (shox 2 ) overexpression-associated reduction of extracellular matrix components; however, it did not inhibit the increase in matrix metalloproteinase (MMP)9 and MMP13, or apoptosis in the temporomandibular joint, which suggests that overexpression of Ihh only partially inhibits shox 2 overexpression-associated congenital dysplasia of the temporomandibular joint (6). Dysregulation of Ihh signaling results in multiple bone diseases, including progressive osseous heteroplasia (7). Treatment with an Ihh signaling inhibitor has been reported to reduce the occurrence of chondroma-like lesions, including enchondromas and osteochondromas, adjacent to disordered growth plates in fibroblast growth factor receptor 3-deficient mice (8). Ihh deletion also induces symphalangism, characterized by initial cartilaginous fusion preventing epiphyseal growth plate formation, resulting in abnormal directionality of chondrocyte differentiation in mutant mice (9). Ihh signaling serves an important role in the mineralization process of fibrocartilaginous entheses (10). Inhibiting $\mathrm{Hh}$ signaling reduces mineralized fibrocartilage, leading to less collagen embedded within mineralized tissue (11). Understanding the signaling mechanisms and functions of Ihh signaling in bone development may provide important insights into bone disease prevention and therapeutics. 
In the present study, Ihh was knocked down in chondrocytes using short hairpin (sh)RNA to investigate the function of Ihh signaling in chondrocyte proliferation and differentiation. The present study also aimed to explore the potential mechanism by which Ihh induces chondrocyte apoptosis and cell cycle arrest.

\section{Materials and methods}

Cell line and cell transfection. Mouse chondrocyte cells (CP-M087) were purchased from Procell Life Science \& Technology Co., Ltd. (Wuhan, China). Cells were grown routinely in Dulbecco's modified Eagle's medium/F-12 medium (Invitrogen; Thermo Fisher Scientific, Inc., Waltham, MA, USA) supplemented with $10 \%$ fetal bovine serum (Gibco; Thermo Fisher Scientific, Inc.) and cultured for $24 \mathrm{~h}$ before refreshing the medium in a $37^{\circ} \mathrm{C}$ humidified atmosphere containing $5 \% \mathrm{CO}_{2}$. Knockdown of Ihh in chondrocyte cells was achieved by transfection with $5 \mu \mathrm{l}\left(10^{9} \mathrm{U}\right)$ lentivirus containing Ihh shRNA (Ihh-shRNA; Shanghai GenePharma Co., Ltd., Shanghai, China) for $48 \mathrm{~h}$ using Lipofectamine 3000 (Thermo Fisher Scientific, Inc.) according to the manufacturer's protocol. Cells transfected with empty lentivirus were used as a negative control (NC), and untreated cells were used as blank control. Cells $\left(5 \times 10^{5}\right.$ cells $\left./ \mathrm{ml}\right)$ were seeded in 6 -well clusters $(1 \mathrm{ml})$ or 96 -well plates $(0.2 \mathrm{ml})$ and transfected for $48 \mathrm{~h}$. Transfected cells were used in further assays or RNA/protein extraction.

RNA extraction and reverse transcription-quantitative polymerase chain reaction $(R T-q P C R)$. Total RNA was extracted from cells using TRIzol reagent (Invitrogen; Thermo Fisher Scientific, Inc.) according to the manufacturer's protocol. RevertAid First Strand cDNA Synthesis Kit (Thermo Fisher Scientific, Inc.) was used to reverse transcribe the mRNA to cDNA according to the manufacturer's protocol. Briefly, $0.5 \mathrm{ng}$ of the template RNA, $1 \mu$ l oligo(dT)18 primer and $12 \mu 1$ RNase free water were mixed, and incubated at $65^{\circ} \mathrm{C}$ for $5 \mathrm{~min}$, then cooled down on ice. The mixture was added to Reaction Buffer (4 $\mu \mathrm{l})$, RiboLock RNase inhibitor (1 $\mu \mathrm{l})$, $10 \mathrm{mM}$ dNTP mix $(2 \mu \mathrm{l})$ and RevertAid Reverse Transcriptase $(1 \mu \mathrm{l})$, and incubated at $42^{\circ} \mathrm{C}$ for $1 \mathrm{~h}$. Finally, the mixture was heated to $70^{\circ} \mathrm{C}$ for $5 \mathrm{~min}$ to obtain the cDNA. Ihh, parathyroid hormone-related protein (PTHrP), transforming growth factor (TGF)- $\beta$, osteocalcin (OCN), runt-related transcription factor 2 (Runx2), osteoprotegerin (OPG), mothers against decapentaplegic homolog 2 (Smad2), Smad3, collagen type $\mathrm{X} \alpha 1$ chain (COL10A) and receptor activator of nuclear factor- $\kappa \mathrm{B}$ ligand (RANKL) mRNA expression was measured using SYBR Fast qPCR Mix (cat. no. RR430A; Takara Biotechnology Co., Ltd., Dalian, China) under the following conditions: $95^{\circ} \mathrm{C}$ for $30 \mathrm{sec}$, followed by 40 cycles of $95^{\circ} \mathrm{C}$ for $5 \mathrm{sec}$ and $60^{\circ} \mathrm{C}$ for $10 \mathrm{sec}$, and then for melting curves (one cycle of $95^{\circ} \mathrm{C}$ for $5 \mathrm{sec}$ and $60^{\circ} \mathrm{C}$ for $60 \mathrm{sec}$, followed by cooling to $50^{\circ} \mathrm{C}$ in $30 \mathrm{sec}$ ) $\beta$-actin was used as an endogenous control. Primers sequences used for qPCR were as follows: Ihh, forward 5'-CTCAGCCTG CTCTCACTACG-3' and reverse 5'-AAGCACATCCAACCC ACCTC-3'; PTHrP, forward 5'-CGAGGTTCAAAGGTTTGC CTC-3' and reverse 5'-GGCCAGAGAAGCCTGTTACC-3'; TGF- $\beta$, forward 5'-AGGGCTACCATGCCAACTTC-3' and reverse 5'-TGACACAGAGATCCGCAGTC-3'; OCN, forward
5'-TCCTTTGGGGTTTGGCCTAC-3' and reverse 5'-CTT GGACACAAAGGCTGCAC-3'; RUNX2, forward 5'-CGC CTCACAAACAACCACAG-3' and reverse 5'-TCACTGTGC TGAAGAGGCTG-3'; OPG, forward 5'-CTGGAACCCCAG AGCGAAAT-3' and reverse 5'-GCGTTTACTTTGGTGCCA GG-3'; Smad2, forward 5'-GTTCCTTTCCTCCTCCGCTC-3' and reverse 5'-AGTCTCTTCACAACTGGCGG-3'; Smad3, forward 5'-TTCACTGGTGCTGGGGTTAG-3' and reverse 5'-GGTAGGGATTCACGCAGACC-3'; COL10A, forward 5'-CTCCCAGCACGCAGAATC-3' and reverse: 5'-TTCCCT ACAGCTGATGGTCC-3'; RANKL, forward 5'-GGAGTT GGCCGCAGACAAGA-3' and reverse 5'-TGATGTGCTGTG ATCCAACGA-3'; and $\beta$-actin, forward 5'-GCAGGAGTA TGACGAGTCCG-3' and reverse 5'-AACAACGCATCT CATATTTGGAA-3'. Data were processed using the $2^{-\Delta \Delta C q}$ method (12).

Cell Counting Kit-8 (CCK-8) cell proliferation assay. Cell proliferation rates were measured using CCK-8 (Beyotime Institute of Biotechnology, Haimen, China). A total of $0.5 \times 10^{4}$ cells were seeded in 96 -well plates for $24 \mathrm{~h}$, transfected with the indicated lentivirus, and further incubated for 24,48 and $72 \mathrm{~h}$ at $37^{\circ} \mathrm{C}$, respectively. A total of $10 \mu \mathrm{lCCK}-8$ reagents were added to each well at $1 \mathrm{~h}$ prior to the endpoint of incubation (23, 47 and $71 \mathrm{~h})$. The optical density of each well was subsequently determined using a microplate reader at a wavelength of $490 \mathrm{~nm}$.

Flow cytometric analysis of apoptosis with Annexin-V/propidium iodide (PI) double staining. An Annexin V Apoptosis Detection kit (Thermo Fisher Scientific, Inc.) was used to assess apoptosis. Following incubation for 24,48 or $72 \mathrm{~h}$ at $37^{\circ} \mathrm{C}$, the cells were trypsinized for $2 \mathrm{~min}$ at $37^{\circ} \mathrm{C}$, collected by centrifugation $(960 \mathrm{x} \mathrm{g})$ for $5 \mathrm{~min}$ at room temperature and resuspended in $2 \mathrm{ml}$ medium. Approximately $2 \times 10^{5}$ cells were harvested, washed twice with cold PBS and resuspended in $500 \mu \mathrm{l}$ binding buffer provided in the kit. A total of $10 \mu \mathrm{l}$ Annexin V-fluorescein isothiocyanate and $10 \mu \mathrm{l}$ PI was added to the solution and mixed well. Following incubation for $15 \mathrm{~min}$ at $37^{\circ} \mathrm{C}$, cells were analyzed using a flow cytometer (BD Biosciences, San Jose, CA, USA).

Cell cycle analysis assay. Cell cycle analysis was determined by flow cytometry using a Cell Cycle and Apoptosis Analysis kit (cat no. C1052, Beyotime Institute of Biotechnology) and analyzed the data using Flowjo (7.6 version; Flowjo LLC, Ashland, OR, USA). Briefly, $\sim 1 \times 10^{4}$ cells were seeded in each well of a 6-well plate. Following $48 \mathrm{~h}$ transfection with Ihh-shRNA or empty lentivirus, cells were harvested and fixed in $70 \%$ ice-cold ethanol for $24 \mathrm{~h}$, followed by staining with PI for $10 \mathrm{~min}$ at room temperature. The different phases of the cell cycle were analyzed using a FACSCalibur flow cytometer (BD Biosciences).

Alkaline phosphatase (ALP) assay. Alkaline Phosphatase Assay Kit (cat no. P0321, Beyotime Institute of Biotechnology) was used to detect ALP activity according to the manufacturer's protocol. Black cobaltous sulfide staining indicated a positive signal. Cells were counterstained with nuclear fast red for $5 \mathrm{~min}$ at room temperature to label the nucleus and 
cytoplasm of cells. Images were captured using an inverted microscope (magnification, x100; Nikon Eclipse TC 100; Nikon Corporation, Tokyo, Japan).

Determination of mineralization using von Kossa staining. Von Kossa staining (Sigma-Aldrich; Merck KGaA, Darmstadt, Germany) was used to determine the extent of minerals deposited on chondrocyte cells. Silver nitrate solution was added to the cells and the plate was exposed to ultraviolet light for 30 min. Cells were subsequently washed with PBS and the reaction was stopped with the addition of $500 \mu \mathrm{l}$ of $5 \%$ sodium thiosulfate (Sigma-Aldrich; Merck KGaA). The positive mineral depositions were stained in black. Cells were counterstained with nuclear fast red for $5 \mathrm{~min}$ at room temperature to label the nucleus and cytoplasm. Images were captured using an inverted microscope (magnification, x100; Nikon Eclipse TC 100; Nikon Corporation).

Western blot analysis. Immunoblotting was performed to detect the expression of Ihh, PTHrP, TGF- $\beta$, OCN, Runx 2 , OPG, Smad2, Smad3, COL10A and RANKL in chondrocyte cells. Cultured or transfected cells were lysed in radioimmunoprecipitation assay buffer (Wuhan Boster Biological Technology, Ltd., Wuhan, China) with $1 \%$ phenylmethylsulfonyl fluoride. The concentration of protein was determined using the BCA Protein Assay kit (cat no. P0011; Beyotime Institute of Biotechnology, Haimen, China) according to the manufacturer's protocol. A total of $60 \mu \mathrm{g} /$ lane of proteins were separated by $12 \%$ SDS-PAGE and transferred onto polyvinyl difluoride membranes. The membranes were blocked in non-fat milk for $1 \mathrm{~h}$ at $37^{\circ} \mathrm{C}$. Membranes were incubated at $4^{\circ} \mathrm{C}$ overnight with the following primary antibodies: Anti-Ihh, (cat. no. SAB1403965; dilution, 1:1,000); anti-PTHrP (cat. no. SAB5300029; dilution, 1:1,000); anti-TGF- $\beta$ (cat. no. SAB4502958; dilution, 1:2,000); anti-OCN (cat. no. SAB1306277; dilution, 1:2,000; all from Sigma Aldrich; Merck KGaA); anti-RUNX2 (cat. no. 12556; dilution, 1:1,000); anti-OPG (cat. no. 4816; dilution, 1:1,000); anti-Smad 2 (cat. no. 5339; dilution, 1:1,000), anti-Smad3 (cat. no. 9523; dilution, 1:1,000; all from Cell Signaling Technology, Inc., Danvers, MA, USA); anti-COL10A (cat. no. ab58632; dilution, 1:1,000); anti-RANKL (cat. no. ab9957; dilution, 1:1,000) and anti-GAPDH (cat. no. ab9485; dilution, 1:3,000; all from Abcam, Cambridge, UK). The blots were subsequently incubated with goat anti-rabbit IgG (cat. no. ab7090) and goat anti-mouse (cat. no. ab97040; both 1:5,000; Abcam) horseradish peroxidase-conjugated secondary antibodies for $1 \mathrm{~h}$ at $37^{\circ} \mathrm{C}$. Signals were visualized using enhanced chemiluminescence substrates (EMD Millipore, Billerica, MA, USA). The densitometry of bands was analyzed using Quantity One software (version 4.6.9; Bio-Rad Laboratories, Inc., Hercules, CA, USA). GAPDH was used as an endogenous protein for normalization.

Statistical analysis. All data are expressed as the mean \pm standard deviation of three independent experiments. Statistical analyses were performed using SPSS v. 17.0 (SPSS, Inc., Chicago, IL, USA). Differences between groups were analyzed using Student's t-test or one-way analysis of variance with Tukey's post hoc test dependent on the conditions. $\mathrm{P}<0.05$ was considered to indicate a statistically significant difference.

\section{Results}

Effects of Ihh on chondrocyte cell proliferation, apoptosis and cell cycle. To investigate the biological role of Ihh in chondrocyte cell growth, chondrocytes transfected with Ihh shRNA were used in the present study. The results of RT-qPCR revealed that Ihh expression was significantly reduced in transfected cells compared with NC cells ( $\mathrm{P}<0.001$; Fig. 1A). Ihh downregulation significantly inhibited cell growth $(\mathrm{P}<0.05$; Fig. 1B) and significantly increased the apoptosis rate compared with the $\mathrm{NC}$ group $(\mathrm{P}<0.001$; Fig. $2 \mathrm{~A})$. In addition, Ihh knockdown significantly reduced the percentage of cells in $\mathrm{S}$ phase $(\mathrm{P}<0.05)$ and increased the percentage of cells in $\mathrm{G} 1$ phase compared with the $\mathrm{NC}$ group $(\mathrm{P}<0.01)$, indicating cell cycle arrest at $\mathrm{G} 1$ to $\mathrm{S}$ phase (Fig. 2B).

Effects of Ihh on chondrocyte cell differentiation. ALP is an enzyme that is required for bone mineralization (13). ALP activity was assessed in the present study and was markedly decreased in Ihh knockdown cells compared with the NC group (Fig. 3A). The calcification and mineralization of bone matrix are essential for the strength and rigidity of the spinal skeletal system (14). To estimate mineralization and calcification, von Kossa staining was performed following Ihh knockdown. Representative images of von Kossa staining were obtained by bright field microscopy (Fig. 3B). Compared with the NC cells, mineral deposition was markedly decreased in the Ihh-shRNA group.

Effects of Ihh on TGF- $\beta /$ Smads and OPG/RANKL signaling. The effects of Ihh on TGF- $\beta /$ Smads and OPG/RANKL signaling were also investigated. The mRNA expression of COL10A, RANKL, Samd2 and Smad3 was significantly upregulated in the Ihh-shRNA group compared with the NC cells $(\mathrm{P}<0.001$; Fig. 4), whereas the levels of OCN, OPG, PTHrP, Runx 2 and TGF- $\beta$ were significantly downregulated in the Ihh-shRNA compared with the NC group $(\mathrm{P}<0.001$; Fig. 4). Protein expression of these genes was further assessed by western blot analysis (Fig. 5). The protein expression of COL10A, RANKL, Samd2 and Smad3 were upregulated, whereas levels of OCN, OPG, PTHrP, Runx 2 and TGF- $\beta$ were significantly downregulated in Ihh knockdown cells compared with the NC group $(\mathrm{P}<0.05$; Fig. 5). These findings suggest that the inhibitory effects of Ihh knockdown on bone differentiation and generation are achieved via the TGF- $\beta /$ Smads and OPG/RANKL signaling pathway.

\section{Discussion}

In the present study, Ihh knockdown was demonstrated to significantly inhibit cell growth and increase the apoptosis rate compared with NC cells. Downregulation of Ihh resulted in cell cycle arrest at G1 to S phase in chondrocytes. In addition, Ihh knockdown decreased the ALP activity and mineral deposition of chondrocytes. These results suggest that the inhibitory roles of Ihh downregulation on chondrocyte growth and differentiation may be associated with the TGF- $\beta / S m a d s$ and OPG/RANKL signaling pathways.

Ihh signaling is essential for chondrocyte differentiation and endochondral ossification (15). Deletion of Ihh in postnatal 

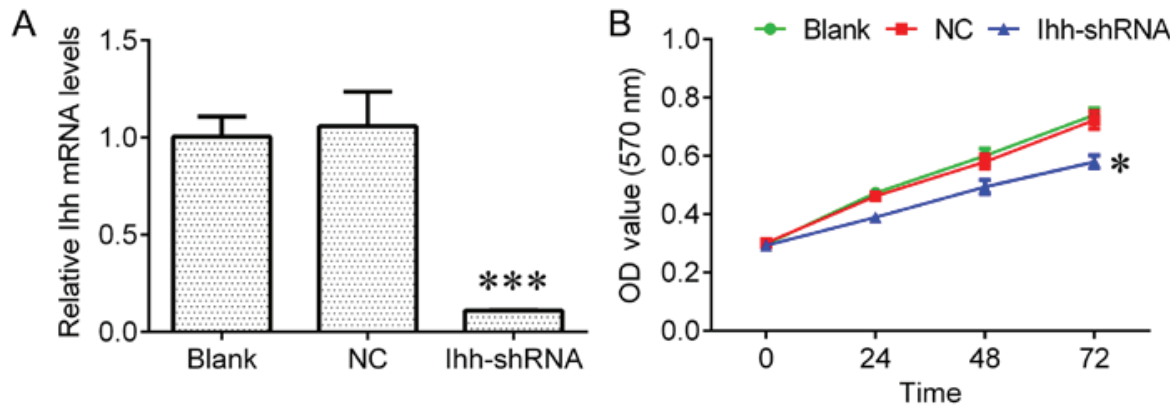

Figure 1. (A) Reverse transcription-quantitative polymerase chain reaction was used to detect Ihh expression and (B) Cell Counting Kit-8 was used to assess cell viability in chondrocytes following transfection with Ihh shRNA or empty lentivirus. ${ }^{*} \mathrm{P}<0.05$ and ${ }^{* * *} \mathrm{P}<0.001$ vs. NC. Ihh, Indian hedgehog; shRNA, short hairpin RNA; NC, negative control; OD, optical density.

A
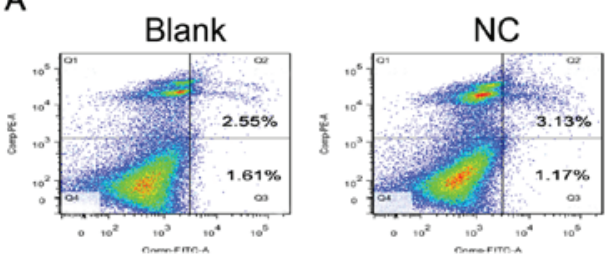

B Blank

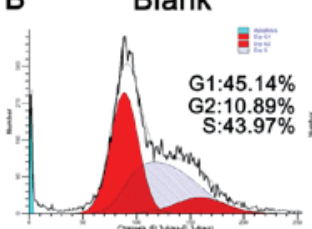

NC

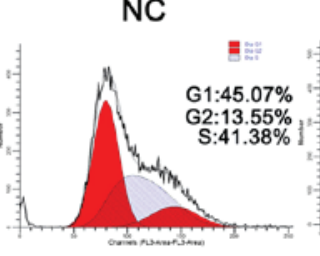

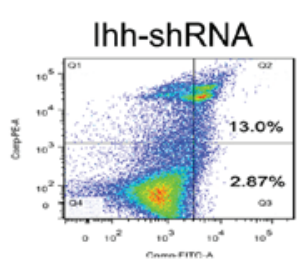

Ihh-ShRNA

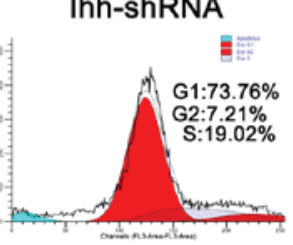

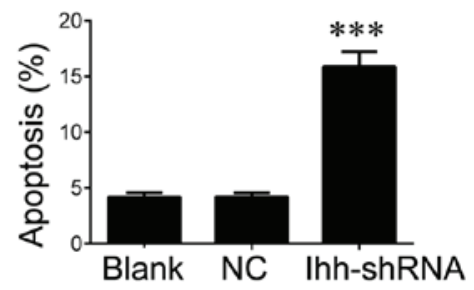

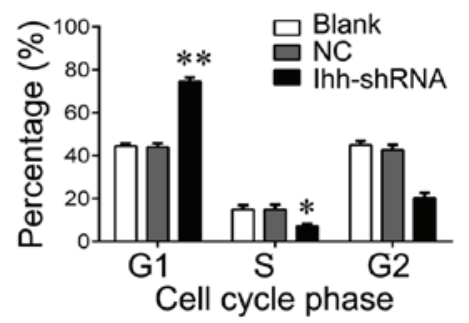

Figure 2. Chondrocytes were transfected with Ihh shRNA or empty lentivirus. (A) Apoptosis and (B) cell cycle distribution were assessed using flow cytometry. ${ }^{*} \mathrm{P}<0.05,{ }^{* *} \mathrm{P}<0.01$ and ${ }^{* * *} \mathrm{P}<0.001$ vs. NC. Ihh, Indian hedgehog; shRNA, short hairpin RNA; NC, negative control.

chondrocytes in a temporal/spatial-specific manner results in the loss of ectopic hypertrophic chondrocyte formation in the growth plate (16). In a previous study, mutant mice with Ihh deletion exhibited a continuous loss of trabecular bone over time, which indicates that postnatal chondrocyte-derived Ihh is essential for skeletal growth, maintaining the growth plate and sustaining trabecular bone growth (16). A previous report demonstrated that treating chondrogenic cells with an Ihh inhibitor inhibited chondrocyte differentiation and secondary ossification center formation (17). Furthermore, Gualeni et al (18) previously demonstrated that diastrophic dysplasia mouse proteoglycan undersulfation resulted in reduced chondrocyte proliferation in the proliferative zone via the Ihh pathway, contributing to reduced long bone growth. Ihh expression has been reported to be significantly increased in humans with the modic degeneration I and II groups and is positively correlated with the severity of degeneration (19). Overexpression of Ihh signaling promotes abnormal chondrocyte differentiation in endochondral ossification and enhances bone formation in posterior longitudinal ligaments (20). Ihh is synthesized by chondrocytes and is required for the synthesis of PTHrP (21). Ihh acts with PTHrP in a negative feedback loop to regulate early chondrocyte differentiation and hypertrophic differentiation (22). Blocking Ihh signaling with cyclopamine has been reported to delay chondrocyte hypertrophy in PTHrP
A

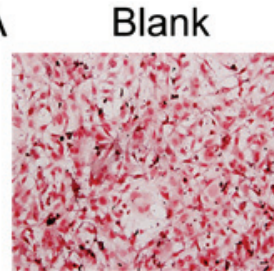

NC

Ihh-shRNA

B

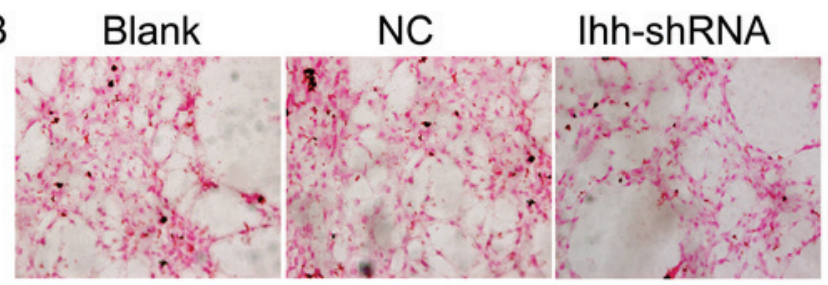

Figure 3. Alkaline phosphatase (ALP) assay was used to determine alkaline phosphatase activity in chondrocytes. (A) Alkaline phosphatase activity was markedly decreased in chondrocytes transfected with Ihhsh RNA compared with the NC group. Magnification, x100. (B) Von Kossa staining was used to determine the extent of mineral deposition (black staining) in chondrocyte cells. Deposition was markedly decreased in cells transfected with Ihh-shRNA compared with the NC group. Ihh, Indian hedgehog; shRNA, short hairpin RNA; NC, negative control.

knockout embryos, whereas upregulating Ihh signaling in the postnatal cartilage led to accelerated chondrocyte hypertrophy 


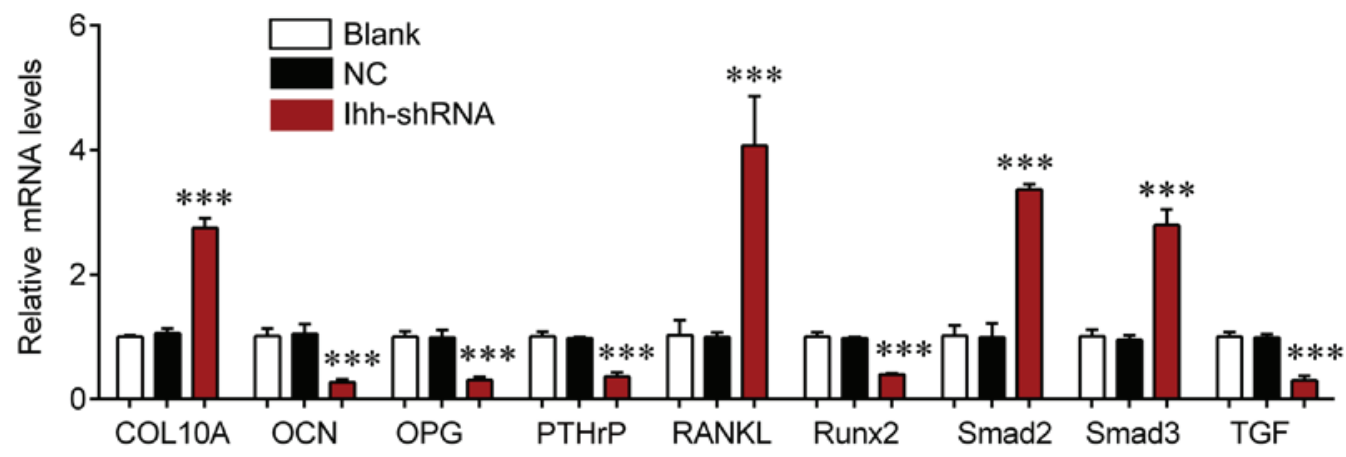

Figure 4. Reverse transcription-quantitative polymerase chain reaction was used to detect the mRNA expression of COL10A, RANKL, Smad2, Smad3, OCN, OPG, PTHrP, Runx 2 and TGF- $\beta$ in chondrocytes following transfection with Ihh shRNA or empty lentivirus. ${ }^{* * *} \mathrm{P}<0.001$ vs. NC. COL10A, collagen type X $\alpha 1$ chain; RANKL, receptor activator of nuclear factor- $\mathrm{kB}$ ligand; Smad, mothers against decapentaplegic; OCN, osteocalcin; OPG, osteoprotegerin; PTHrP, parathyroid hormone-related protein; Runx2, runt-related transcription factor 2; TGF, transforming growth factor; Ihh, Indian hedgehog; shRNA, short hairpin RNA; NC, negative control.

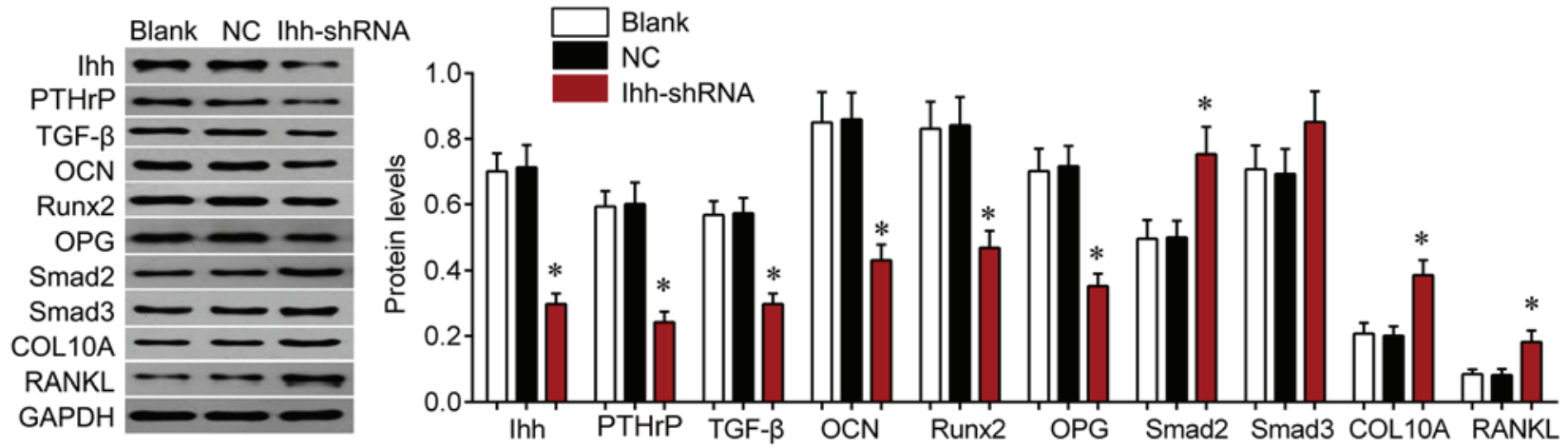

Figure 5. Western blot analysis was used to detect the protein expression of COL10A, RANKL, Smad2, Smad3, OCN, OPG, PTHrP, Runx2 and TGF- $\beta$ in chondrocytes following transfection with Ihh shRNA or empty lentivirus. " $\mathrm{P}<0.05$ vs. NC. COL10A, collagen type X $\alpha 1$ chain; RANKL, receptor activator of nuclear factor- $\mathrm{kB}$ ligand; Smad, mothers against decapentaplegic homolog; OCN, osteocalcin; OPG, osteoprotegerin; PTHrP, parathyroid hormone-related protein; Runx2, runt-related transcription factor 2; TGF, transforming growth factor; Ihh, Indian hedgehog; shRNA, short hairpin RNA; NC, negative control.

during secondary ossification, indicating that Ihh signaling promotes chondrocyte hypertrophy independently of PTHrP, which may be mediated by Wnt/ $\beta$-catenin signaling $(23,24)$. As chondrocytes go through a program of proliferation and subsequent differentiation into hypertrophic chondrocytes, PTHrP maintains chondrocyte proliferation and delays their further differentiation (25). The differentiation-delaying action of PTHrP is mediated by suppressing the synthesis of Runx2, which is a transcription factor integral to osteoblast differentiation (26). In the absence of Ihh, the osteoblast fails to activate the expression of Runx2 (27). However, forced expression of Runx 2 in the skeletogenic cells restores bone formation in the Runx2-null embryo, whereas it does not in the Ihh-null embryo; this suggests that Ihh-induced osteoblast differentiation requires additional effectors (27).

The results of the present study demonstrate that the mRNA and protein expression of hypertrophic markers, including COL10A, RANKL, Smad2 and Smad3, are upregulated in chondrocytes following Ihh knockdown, whereas levels of OCN, OPG, PTHrP Runx 2 and TGF- $\beta$ are significantly reduced. Previous studies have reported that multiple signaling pathways, including Wnt/ $\beta$-catenin and TGF- $\beta / \mathrm{Smad}$ pathways, are able to regulate chondrocyte hypertrophy (28). The Smad2/3 pathway is directly activated by TGF- $\beta$, which leads to inhibited hypertrophy (29). TGF- $\beta$ activation of Smad3 also inhibits Runx2 via epigenetic regulation (30). Glioma-associated oncogene-Krüppel family members (Gli) 1 and 2, which are Ihh downstream transcription factors, increase COL10A activity and Runx 2 promotes COL10A1 expression via interacting with Ihh (31). Furthermore, Gli1 and Gli2 act in a complex with Runx2/Smad to induce chondrocyte differentiation (30), suggesting that Ihh signaling may be an important factor for early chondrocyte differentiation and the maturation and calcification of chondrocytes.

In conclusion, knockdown of Ihh suppresses chondrocyte growth and differentiation and this effect may be associated with the TGF- $\beta /$ Smad and OPG/RANKL signaling pathways. These results suggest that chondrocyte-derived Ihh is essential for maintaining the growth plate and that manipulating Ihh expression or its signaling components may be a novel effective treatment for achondroplasia and other skeletal diseases.

\section{Acknowledgements}

The present study was supported by the Natural Science Foundation of Hunan Province (grant no. 2016JJ3160) and the National Natural Science Foundation of China (grant no. 81472145$)$. 


\section{References}

1. Wang T, Zhang X and Bikle DD: Osteogenic differentiation of periosteal cells during fracture healing. J Cell Physiol 232: 913-921, 2017.

2. Komori T: Cell death in chondrocytes, osteoblasts, and osteocytes. Int J Mol Sci 17: pii: E2045, 2016.

3. Xu J, Li Z, Hou Y and Fang W: Potential mechanisms underlying the Runx 2 induced osteogenesis of bone marrow mesenchymal stem cells. Am J Transl Res 7: 2527-2535, 2015.

4. Day TF and Yang Y: Wnt and hedgehog signaling pathways in bone development. J Bone Joint Surg Am 90 (Suppl 1): S19-S24, 2008.

5. Cai H and Liu A: Spop promotes skeletal development and homeostasis by positively regulating Ihh signaling. Proc Natl Acad Sci USA 113: 14751-14756, 2016.

6. Li X, Liang W, Ye H, Weng X, Liu F, Lin $P$ and Liu X: Overexpression of Indian hedgehog partially rescues short stature homeobox 2-overexpression-associated congenital dysplasia of the temporomandibular joint in mice. Mol Med Rep 12: 4157-4164, 2015.

7. Yang J, Andre P, Ye L and Yang YZ: The hedgehog signalling pathway in bone formation. Int J Oral Sci 7: 73-79, 2015.

8. Zhou S, Xie Y, Tang J, Huang J, Huang Q, Xu W, Wang Z, Luo F, Wang Q, Chen H, et al: FGFR3 deficiency causes multiple chondroma-like lesions by upregulating hedgehog signaling. PLoS Genet 11: e1005214, 2015.

9. Amano K, Densmore M, Fan Y and Lanske B: Ihh and PTH1R signaling in limb mesenchyme is required for proper segmentation and subsequent formation and growth of digit bones. Bone 83: 256-266, 2016.

10. Bechtold TE, Saunders C, Decker RS, Um HB, Cottingham N, Salhab I, Kurio N, Billings PC, Pacifici M, Nah HD and Koyama E: Osteophyte formation and matrix mineralization in a TMJ osteoarthritis mouse model are associated with ectopic hedgehog signaling. Matrix Biol 52-54: 339-354, 2016.

11. Breidenbach AP, Aschbacher-Smith L, Lu Y, Dyment NA, Liu CF, Liu H, Wylie C, Rao M, Shearn JT, Rowe DW, et al: Ablating hedgehog signaling in tenocytes during development impairs biomechanics and matrix organization of the adult murine patellar tendon enthesis. J Orthop Res 33: 1142-1151, 2015.

12. Livak KJ and Schmittgen TD: Analysis of relative gene expression data using real-time quantitative PCR and the 2(-Delta Delta C(T)) method. Methods 25: 402-408, 2001.

13. Orimo $\mathrm{H}$ : The mechanism of mineralization and the role of alkaline phosphatase in health and disease. J Nippon Med Sch 77: 4-12, 2010

14. Fujisawa R and Tamura M: Acidic bone matrix proteins and their roles in calcification. Front Biosci (Landmark Ed) 17: 1891-1903, 2012.

15. Chen X, Macica CM, Nasiri A and Broadus AE: Regulation of articular chondrocyte proliferation and differentiation by indian hedgehog and parathyroid hormone-related protein in mice. Arthritis Rheum 58: 3788-3797, 2008.

16. Maeda Y, Nakamura E, Nguyen MT, Suva LJ, Swain FL, Razzaque MS, Mackem S and Lanske B: Indian hedgehog produced by postnatal chondrocytes is essential for maintaining a growth plate and trabecular bone. Proc Natl Acad Sci USA 104: 6382-6387, 2007.

17. Xing W, Cheng S, Wergedal J and Mohan S: Epiphyseal chondrocyte secondary ossification centers require thyroid hormone activation of Indian hedgehog and osterix signaling. J Bone Miner Res 29: 2262-2275, 2014.
18. Gualeni B, Facchini M, De Leonardis F, Tenni R, Cetta G, Viola M, Passi A, Superti-Furga A, Forlino A andRossi A: Defective proteoglycan sulfation of the growth plate zones causes reduced chondrocyte proliferation via an altered Indian hedgehog signalling. Matrix Biol 29: 453-460, 2010.

19. Sugita D, Yayama T, Uchida K, Kokubo Y, Nakajima H, Yamagishi A, Takeura $\mathrm{N}$ and Baba H: Indian hedgehog signaling promotes chondrocyte differentiation in enchondral ossification in human cervical ossification of the posterior longitudinal ligament. Spine (Phila Pa 1976) 38: E1388-E1396, 2013.

20. Kobayashi T, Chung UI, Schipani E, Starbuck M, Karsenty G, Katagiri T, Goad DL, Lanske B and Kronenberg HM: PTHrP and Indian hedgehog control differentiation of growth plate chondrocytes at multiple steps. Development 129: 2977-2986, 2002.

21. Zhou J, Wei X and Wei L: Indian hedgehog, a critical modulator in osteoarthritis, could be a potential therapeutic target for attenuating cartilage degeneration disease. Connect Tissue Res 55: 257-261, 2014

22. Mak KK, Kronenberg HM, Chuang PT, Mackem S and Yang Y: Indian hedgehog signals independently of PTHrP to promote chondrocyte hypertrophy. Development 135: 1947-1956, 2008.

23. Brechbiel JL, Ng JM and Curran T: PTHrP treatment fails to rescue bone defects caused by hedgehog pathway inhibition in young mice. Toxicol Pathol 39: 478-485, 2011.

24. Minina E, Wenzel HM, Kreschel C, Karp S, Gaffield W, McMahon AP and Vortkamp A: BMP and Ihh/PTHrP signaling interact to coordinate chondrocyte proliferation and differentiation. Development 128: 4523-4534, 2001.

25. Kronenberg HM: PTHrP and skeletal development. Ann N Y Acad Sci 1068: 1-13, 2006.

26. Tu X, Joeng KS and Long F: Indian hedgehog requires additional effectors besides Runx2 to induce osteoblast differentiation. Dev Biol 362: 76-82, 2012

27. Studer D, Millan C, Öztürk E, Maniura-Weber K and Zenobi-Wong M: Molecular and biophysical mechanisms regulating hypertrophic differentiation in chondrocytes and mesenchymal stem cells. Eur Cell Mater 24: 118-135, 2012.

28. Kim KO, Sampson ER, Maynard RD, O'Keefe RJ, Chen D, Drissi H, Rosier RN, Hilton MJ and Zuscik MJ: Ski inhibits TGF- $\beta /$ phospho-Smad3 signaling and accelerates hypertrophic differentiation in chondrocytes. J Cell Biochem 113: 2156-2166, 2012.

29. Kang JS, Alliston T, Delston R and Derynck R: Repression of Runx 2 function by TGF-beta through recruitment of class II histone deacetylases by Smad3. EMBO J 24: 2543-2555, 2005.

30. Shimoyama A, Wada M, Ikeda F, Hata K, Matsubara T, Nifuji A, Noda M, Amano K, Yamaguchi A, Nishimura R and Yoneda T: Ihh/Gli2 signaling promotes osteoblast differentiation by regulating Runx2 expression and function. Mol Biol Cell 18: 2411-2418, 2007.

31. Amano K, Densmore M, Nishimura R and Lanske B: Indian hedgehog signaling regulates transcription and expression of collagen type X via Runx2/Smads interactions. J Biol Chem 289: 24898-24910, 2014.

This work is licensed under a Creative Commons Attribution-NonCommercial-NoDerivatives 4.0 International (CC BY-NC-ND 4.0) License. 